\title{
SÍNDROME DE TOLOSA-HUNT
}

\section{DIFICULDADES NO DIAGNÓSTICO E PADRÃO DE RESPOSTA À PREDNISONA}

\author{
PAULO EDUARDO MESTRINELLI CARRILHO*, FÁBIO IUII YAMAMOTO**, MILBERTO SCAFF***
}

\begin{abstract}
RESUMO - A síndrome de Tolosa-Hunt (STH) consiste de oftalmoplegia dolorosa relacionada a processo inflamatório granulomatoso no seio cavernoso. Seu diagnóstico só pode ser firmado quando outras causas potenciais de oftalmoplegia dolorosa forem descartadas. Resposta satisfatória e padronizada a corticosteróides, associada a evolução benigna, pode reforçar essa possibilidade, embora pseudotumor orbitário e neoplasias do tipo linfoma e até meningioma possam suscitar dúvidas no diagnóstico diferencial, pois também podem apresentar boa resposta a essa medicação. Faz-se necessário, portanto, ampla investigação complementar. Apresentamos estudo clínico-radiológico-laboratorial de cinco pacientes com STH. Prednisona foi utilizada em doses entre 40 e $80 \mathrm{mg} /$ dia. Houve resposta analgésica excelente, com remissão da dor em menos de 48 horas em 4 pacientes. Houve melhora mais lenta da oftalmoplegia, que regrediu completamenteentre 4 e 45 dias em todos os pacientes.
\end{abstract}

PALAVRAS-CHAVE: síndrome de Tolosa Hunt, oftalmoplegia, doenças orbitárias, prednisona.

Tolosa-Hunt syndrome: troubles in diagnosing and pattern of response to prednisone

ABSTRACT - The Tolosa-Hunt syndrome (THS) consists of a painful ophthalmoplegia related to granulomatous inflammatory process in the cavernous sinus. According to recent concepts, the diagnosis is established only when other causes of painful ophthalmoplegia are ruled out. A typical pattern of response to corticosteroids associated with a benign evolution may reinforce this possibility. Tumors such as lymphoma and meningioma and orbital pseudotumors can make difficult the differential diagnosis because they also may respond to steroids. Thus it is always necessary to make an extensive ancillary investigation. We performed a clinical, laboratory and radiologic study of five patients with THS. Prednisone was used in all, with dosages ranging from 40 to $80 \mathrm{mg} / \mathrm{day}$. In four patients there was a dramatic analgesic effect in less than 48 hours. Improvement of the ophthalmoplegia was not so fast but occurred in all with a complete remission in 4 to 45 days.

KEY WORDS: Tolosa-Hunt syndrome, ophthalmoplegia, orbital diseases, prednisone.

Oftalmoplegia dolorosa de caráter inflamatório foi descrita originalmente por Tolosa ${ }^{13} \mathrm{e}$ por Hunt e col. ${ }^{6}$, em 1954 e 1961, respectivamente. Esta entidade, relativamente rara, relaciona-se a processo inflamatório granulomatoso inespecífico do seio cavernoso que tende a cursar para resolução espontânea. Tal evolução seria dramaticamente abreviada por corticóides ${ }^{2,11}$. Para Spector \& Fiandaca ${ }^{12}$ e Hunt \& Brightman ${ }^{5}$, o diagnóstico desta síndrome é baseado na exclusão de outras entidades que cursam com oftalmoplegia dolorosa, principalmente tumores e malformações vasculares intracavernosas, sendo portanto, fundamentais os estudos de imagem na investigação complementar. No entanto, processos do tipo pseudotumor orbital de pequena repercussão clínica

Departamento de Neurologia da Faculdade de Medicina da Universidade de São Paulo (FMUSP): * Médico pós-graduando; ** Mestre em Neurologia, Médico Supervisor da Clínica Neurológica do Hospital das Clínicas da FMUSP, ***Professor Titular. Aceite: 31-outubro-1996. 
podem trazer grande dificuldade diagnóstica. Alguns autores ${ }^{3,10}$ fomentam até a hipótese de ambos serem manifestações regionais polares de uma mesma entidade, já que possuem vários pontos em comum: grande sensiblidade a corticóides, mesmas características radiológicas, inclusive na ressonância nuclear magnética (RNM), além de padrão histológico muito próximo. Além disso, exoftalmia de 2 até 6 milímetros foi observada em $18 \%$ dos pacientes compilados por Kline ${ }^{7}$. Este autor, em 1982, revisou 146 casos da literatura em lingua inglesa relatados até então, dando maior consistência e padronização à entidade descrita por Tolosa e Hunt, a síndrome de Tolosa-Hunt (STH). Dos 47 casos de oftalmoplegia dolorosa atendidos no serviço de neurocirurgia de Reims, entre 1979 e 1990, Rousseaux e col. ${ }^{11}$ firmaram o diagnóstico de STH em apenas sete pacientes, após ampla investigação.

Apresentamos o estudo clínico-laboratorial de cinco pacientes com STH, objetivando detalhar o padrão de resposta clínica ao uso da prednisona.

\section{CASUISTICA E MÉTOdO}

Cinco pacientes com oftalmoplegia dolorosa, preenchendo os critérios estabelecidos por Hunt e col. ${ }^{6} \mathrm{e}$ sistematizados por Hannerz ${ }^{4}$, foram admitidos no Serviço de Neurologia Clínica do Hospital das Clínicas da Universidade de São Paulo, entre 1993 e 1995. Assim sendo, consideramos a STH como entidade caracterizada por dor orbitária episódica associada a paralisia do terceiro, quarto ou sexto nervos cranianos, isoladamente ou em conjunto, que tende a regredir espontaneamente, embora possa ocorrer recidiva e remissão. Critérios adicionais para a STH incluem a duração da oftalmoplegia dolorosa em cerca de 8 semanas se não ocorrer tratamento, melhora em 72 horas mediante uso de corticosteróides e exclusão de outros processos causais através de exames de neuroimagem.

Todos os pacientes foram examinados pelos autores durante a internação hospitalar, assim como acompanhados ambulatorialmente, por período que variou entre 10 e 34 meses.

Tabela 1. Dados clínico-laboratoriais.

\begin{tabular}{llllllll}
\hline Caso S & I Apresentação & $\begin{array}{c}\text { Nervo } \\
\text { craniano }\end{array}$ & TC & RNM & LCR & $\begin{array}{c}\text { Angio Angio TC/RNM } \\
\text { RNM digital órbita }\end{array}$ \\
\hline
\end{tabular}

\begin{tabular}{|c|c|c|c|c|c|c|c|c|c|c|}
\hline JS & $F$ & 46 & dor OE e & $\begin{array}{l}\text { III, VI, V-1 E } \\
\text { diplopia } \\
\text { no d7 }\end{array}$ & $\mathrm{nl}$ & nl & 1 leucócito & $\begin{array}{c}\mathrm{nl} \\
\mathrm{pt}=15 \\
\mathrm{gl}=45\end{array}$ & $\mathrm{nl}$ & - \\
\hline wC & $\mathbf{F}$ & 37 & cefaléia e & $\begin{array}{l}\text { IIIc, IV, V-1 } \\
\text { diplopia } \\
\text { no d15 }\end{array}$ & $\begin{array}{c}n l \\
+V-2 D\end{array}$ & $\mathrm{nl}$ & 12 leucócitos & $\begin{array}{c}\mathrm{nl} \\
\mathrm{pt}=25\end{array}$ & $\mathrm{nl}$ & $\mathrm{nl}$ \\
\hline$A M$ & $F$ & 76 & cefaléia & $\begin{array}{l}\text { III, V-1 D } \\
\text { periorbital D } \\
\text { e diplopia } \\
\text { no d10 }\end{array}$ & $\begin{array}{c}\mathrm{nl} \\
\text { (2 dias } \\
\text { após) }\end{array}$ & $\begin{array}{c}\text { gliose } \\
\text { em SB }\end{array}$ & 1 leucócito & $\begin{array}{c}\mathrm{nl} \\
\mathrm{pt}=22 \\
\mathrm{gl}=63\end{array}$ & - & $\mathrm{nl}$ \\
\hline $\mathrm{MN}$ & $\mathrm{F}$ & 43 & cefaléia e & $\begin{array}{l}\text { IIIc E } \\
\text { diplopia } \\
\text { no dl }\end{array}$ & $\mathrm{nl}$ & $\mathrm{nl}$ & 0,3 leucócitos & $\begin{array}{c}- \\
\mathrm{pt}=21 \\
\mathrm{gl}=65\end{array}$ & $\mathrm{nl}$ & - \\
\hline RS & $F$ & 54 & cefaléia e & $\begin{array}{l}\text { III, IV, V-1 } \\
\text { diplopia } \\
\text { no d3 }\end{array}$ & $\begin{array}{c}\mathrm{nl} \\
+ \\
\mathrm{V}-2 \mathrm{E}\end{array}$ & $\mathrm{nl}$ & 1 leucócito & $\begin{array}{c}\mathrm{nl} \\
\mathrm{pt}=33 \\
\mathrm{gl}=66\end{array}$ & $\mathrm{nl}$ & $\mathrm{nl}$ \\
\hline
\end{tabular}

angio, angiografia; d, dia de evoluçăo; D, direito; E, esquerdo; F, feminino; gl, glicose(mg\%); I, idade (anos); LCR, liquido cefalorraqueano; nl, normal; OE, olho esquerdo; pt, proténa (mg\%); RNM, ressonância nuclear magnética; S, sexo; SB, substância branca; TC, tomografia computadorizada; V-l, ramo oftálmico do trigêmio; V-2, ramo maxilar do trigêmio; IUlc, terceiro nervo completo. 
Tabela 2. Resposta terapêutica à prednisona.

\begin{tabular}{lccc}
\hline Caso & Remissão total da dor & $\begin{array}{c}\text { Remissão total da } \\
\text { oftalmoparesia }\end{array}$ & Recidiva \\
\hline JS & 48 horas & 4 dias & não houve \\
WC & 48 horas & 10 dias & não houve \\
AM & 6 dias & 30 dias & não houve \\
MN & 48 horas & 10 dias & não houve \\
RS & 48 horas & 45 dias & 15 dias após suspensão \\
& & & da medicação
\end{tabular}

O tempo foi estimado a partir do início da terapia. O tempo de remissão foi considerado apenas quando todo e qualquer sinal de oftalmoparesia tivesse desaparecido.

Os seguintes exames complementares foram realizados: análise do líquido cefalorraqueano, tomografia computadorizada (TC) de crânio, RNM de encéfalo, TC ou RNM de órbita, angiografia por RNM e angiografia digital cerebral, além de provas séricas de fase ativa e exames reumatológicos: anticorpos anti-nucleares, fator reumatóide, velocidade de hemossedimentação, mucoproteínas, proteína $\mathrm{C}$ reativa, complemento, eletroforese de proteínas e reaçōes sorológicas para sífilis. Todos os pacientes receberam prednisona por via oral como terapia inicial, na dose única diária estimada de $1 \mathrm{mg} / \mathrm{kg}$. O padrão de resposta foi estudado sob dois aspectos: o efeito analgésico e a regressão da oftalmoplegia.

\section{RESULTADOS}

$\mathrm{Na}$ Tabela 1 estão os achados clínicos e laboratoriais do grupo analisado. A resposta clínica à prednisona é resumida na Tabela 2.

\section{DISCUSSÃO}

Desde a sua descrição, a STH tem sido entidade controversa, sobretudo em relação à sua identidade nosológica e etiológica.

$\mathrm{Na}$ grande revisão de Kline ${ }^{7}$, observou-se que as idades de acometimento variaram de 3 anos e meio a 75 anos, não houve preponderância de sexo, a bilateralidade dos sintomas esteve presente em apenas $5 \%$ dos pacientes e o padrāo de acometimento obedeceu as seguintes frequências: nervo oculomotor: $85 \%$, nervo abducente: $70 \%$, nervo troclear: $29 \%$, nervo trigêmio (ramo oftálmico): $30 \%$. Devemos ressaltar ainda que Hunt e col. ${ }^{6}$ observaram que fibras simpáticas pericarotídeas e o nervo óptico também poderiam ser acometidos. Em menor escala, envolvimento de outros nervos cranianos também pode ocorrer, como o segundo e terceito ramos do nervo trigêmio e os nervos facial e acústico ${ }^{4}$.

Chama a atenção, em nossa casuística, o predomínio absoluto do sexo feminino, contrário aos principais registros da literatura ${ }^{4.6 .7}$, que não citam preponderância de sexo na STH. No entanto, o número de pacientes em nosso estudo é pequeno para que alguma conclusão possa ser firmada a esse respeito.

Atualmente existem mais de 250 casos relatados na literatura e apenas cerca de $10 \%$ foram submetidos a estudo anátomo-patológico ou biópsia da parede do seio cavernoso com estudo histopatológico, nada além de uma reaçāo granulomatosa inespecífica tendo sido observado ${ }^{7,13}$. Em alguns casos havia necrose e células gigantes multinucleadas, levantando a possibilidade de associação com a arterite temporal. Todos os autores são quase unânimes em afirmar que o diagnóstico depende da exclusão de qualquer lesão expansiva ou vascular sediada no seio cavemoso 
e que a boa resposta aos corticóides é a regra, assim como que a evolução clínica é bastante satisfatória, às vezes até independente do uso de drogas esteróides ${ }^{4,5,11,12}$.

O diagnóstico diferencial das oftalmoplegias dolorosas compreende grande número de condições, a saber: oftalmoplegia diabética, aneurismas da artéria comunicante posterior ou da porção intracavernosa da artéria carótida, arterite temporal, lesões expansivas parasselares (adenoma hipofisário, tumor epidermóide, linfoma, sarcoma e metástases), pseudotumor orbitário, doença de Reiter e processos inflamatórios e granulomatosos basais tais como a sarcoidose, aspergilose, actinomicose, sífilis, tuberculose e a granulomatose de Wegener.

Observamos que em todos os nossos pacientes a resposta analgésica à prednisona foi quase dramática: todos tiveram alívio da dor em menos de 48 horas, fato já observado na literatura. Quanto à oftalmoparesia, houve lentidão na resposta ao tratamento, que variou de 4 a 45 dias (média $=19,8$ ). Os nervos cranianos mais acometidos em nossos pacientes obedeceram à seguinte ordem: oculomotor: $100 \%(n=5)$; intrínseco: $40 \%(n=2)$ e extrínseco: $100 \%(n=5)$; trigêmio: $80 \%$ $(n=4)$; V-1: $80 \%(n=4) ;$ V-2: $40 \%(n=2)$; troclear: $40 \%(n=2)$; abducente: $20 \%(n=1)$.

Nesta casuística alguns aspectos são peculiares. A paciente RS apresentou recidiva dos sintomas e sinais 15 dias após a suspensão do corticóide. Tal fato não é incomum, pois vários autores, além do próprio Hunt, já o relataram. Kline ${ }^{7}$ observou recidivas em $39 \%$ dos pacientes (25\% ipsilateralmente e $14 \%$ contralateralmente) e frequência semelhante (33\%) foi relatada por Hannerz ${ }^{4}$. Este autor observou, ainda, em muitos pacientes, sintomas do tipo artralgia, fadiga crônica e equimoses espontâneas, sugerindo que a doença teria aspectos "inflamatórios" sistêmicos. Tais sintomas não foram observados em nosso estudo, porém outros autores ${ }^{14}$ já relataram associação com tireoidite de Hashimoto, reforçando a impressão de uma provável origem "auto-imune" das duas entidades. Deve-se ressaltar que a paciente RS possuía um passado de "tireoidite" e que já havia desenvolvido hipotireoidismo secundário a tal processo.

Quanto à investigação, nenhum achado específico foi observado, mesmo mediante estudo por RNM. Na literatura, porém, vêm sendo relatadas alteraçōes no seio cavernoso, que vāo desde anormalidades de sinal com padrão isointenso ao músculo e hipointenso à gordura em Tl e isointenso à gordura em T2, até alargamento do próprio seio e alteração convexa da sua margem lateral ${ }^{1.15}$. Várias entidades, entretanto, podem cursar com tais características, como por exemplo, o meningioma, o linfoma e a sarcoidose.

Nossos pacientes não foram submetidos à flebografia orbitária. Este exame vem sendo apontado como de utilidade, em 40 a $73 \%$ dos casos, no correto diagnóstico da $\mathrm{STH}^{4,8,9}$, evidenciando desde estreitamento ou irregularidade na veia orbitária superior até oclusão da própria, com presença de circulação colateral. A ocorrência de falsos positivos praticamente inexiste, sendo considerável, entretanto, o número de exames falsos negativos.

Em conclusāo, resposta eficaz a corticosteróides é a regra nos pacientes com STH. Verificamos, com a utilização da prednisona, resposta analgésica excelente e extremamente rápida: houve remissão completa da dor em menos de 48 horas, na maioria dos nossos pacientes. Em contrapartida, a remissão da oftalmoplegia foi mais demorada, tendo ocorrido de 4 a 45 dias após a instituição da terapêutica corticosteróide.

\section{REFERÊNCIAS}

1. Aktan S, Aykut C, Erzen C. Computed tomography and magnetic resonance imaging in three patients with Tolosa-Hunt syndrome. Eur Neurol 1993;33:393-396.

2. Goadsby PJ, Lance JW. Clinicopathological correlation in a case of painful ophthalmoplegia: Tolosa-Hunt syndrome. J Neurol Neurosurg Psychiatry 1989;52:1290-1293.

3. Goto Y, Goto I, Hosokawa S. Neurological and radiological studies in painful ophthalmoplegia: Tolosa-Hunt syndrome and orbital pseudotumor. J Neurol 1989;236:448-451.

4. Hannerz J. Recurrent Tolosa-Hunt syndrome. Cephalalgia 1992;12:45-51. 
5. Hune WE, Brightman RP. The Tolosa-Hunt syndrome: a problem in differential diagnosis. Acta Neurochir 1988;42(Suppl.):248-252.

6. Hunt WE, Meagher JN, LeFever HE, Zeman W. Painful ophthalmoplegia: its relation to indolent inflammation of the cavernous sinus. Neurology 1961;11:56-62.

7. Kline LB. The Tolosa-Hunt syndrome. Surv Ophthalmol 1982;27:79-95

8. Muhletaler CA, Gerlock AJ. Orbital venography in painful ophthalmoplegia ( Tolosa-Hunt). Am J Roetgenol 1979;72:600-616.

9. Nalli DR, Malheiros SMF, Nogueira RG, Abdala N, Caldas JGP, Carrete Jr H, Massaro AR. Avaliaçăo da flebografia orbitária em oito casos de síndrome de Tolosa-Hunt. Arq Neuropsiquiatr 1994;52:231-236.

10. Rosenbaum DH, Davis MJ, Sony IS. The syndrome of painful ophthalmoplegia: a case with intraorbital mass and hypervascularity. Arch Neurol 1979;36:41-43.

11. Rousseaux P, Peruzzi P, Schaison-Cusin M. Le syndrome de Tolosa-Hunt: doit il encore être un diagnostic délimination ?Rev Neurol (Paris) 1993;149:315-319.

12. Spector RH, Fiandaca MD. The "sinister" Tolosa-Hunt syndrome. Neurology 1986;36:198-203.

13. Tolosa E. Periarteritic lesions of the carotid siphon with the clinical features of a carotid infraclinoidal aneurysm. J Neurol Neurosurg Psychiatry 1954;1 17:300-302.

14. Vailati A, Marene C, Comis S, Anstre L, Sozza E, Galende P, Mastignori E, Bossolo PA, Ascari E. Hashimoto's thyroiditis in association with Tolosa-Hunt syndrome: a case report. Thyroid 1993;3:1257.

15. Youssem DM, Atlas SW, Grosman RI, Sergot RC, Savino PS, Bosley TM. MR imaging of Tolosa-Hunt syndrome. ANR 1989;10:1181-1184. 